\title{
O direito à saúde no território: o olhar dos usuários para Atenção Primária à Saúde
}

\author{
The right to health in the territory: \\ service users' perceptions of Primary Health Care
}

Denise da Silva Melo (https://orcid.org/0000-0001-6294-7599) ${ }^{1}$

Ana Lúcia Andrade da Silva (https://orcid.org/0000-0002-2502-7643) ${ }^{2}$

Petrônio José de Lima Martelli (https://orcid.org/0000-0001-6920-6435) ${ }^{2}$

Tereza Maciel Lyra (https://orcid.org/0000-0002-3600-7250) ${ }^{1}$

Gabriella Morais Duarte Miranda (https://orcid.org/0000-0001-9588-6817) ${ }^{2}$

Antônio da Cruz Gouveia Mendes (https://orcid.org/0000-0002-3381-134X) ${ }^{1}$
${ }^{1}$ Instituto Aggeu Magalhães, Fundação Oswaldo Cruz. Av. Prof. Moraes Rego s/n, Cidade Universitária. 50670420 Recife PE Brasil. nyyse.ms@gmail.com ${ }^{2}$ Programa de PósGraduação em Saúde Coletiva, Universidade Federal de Pernambuco. Recife PE Brasil.
Abstract Primary health care is an essential component of effective health systems. The aim of this study aim was to evaluate the quality of primary care in a city in the state of Pernambuco, Brazil. We conducted an exploratory study with 525 service users using structured questionnaires. The quality of primary care was assessed across five dimensions: accessibility, clinical care, professional-user relations, community activities and structure. The findings point to the perpetuation of social vulnerabilities and challenges in achieving equitable universal care. Dissatisfaction rates were highest in the following categories: access to specialist appointments and exams, appointment wait time, and opportunity to make complaints. However, respondents were satisfied with medical and nursing care, particularly in relation to respect, privacy, listening and confidentiality. The findings show that, although health professionals were committed to providing humanized care, fragmentation of care is evident, hampering the provision of adequate and timely follow-up and negatively affecting the quality of care.

Key words Primary health care, Health services research, Patient satisfaction
Resumo A Atenção Primária à Saúde (APS) é considerada imprescindivel para a efetividade dos sistemas de saúde. O objetivo deste estudo foi avaliar a qualidade da assistência prestada na APS em um município de Pernambuco. Realizou-se um estudo exploratório através da escuta a 525 usuários utilizando questionários estruturados. A avaliação da qualidade considerou as dimensões: acessibilidade, assistência clínica, relação profissional-usuário, atividades comunitárias e estrutura. Os resultados apontam para um contexto de perpetuação das vulnerabilidades sociais e o desafio em alcançar um atendimento equitativo e universal. Os principais aspectos de insatisfação foram: dificuldade de acesso a consultas e exames especializados, tempo de espera para atendimento e possibilidade de apresentar reclamações. Por outro lado, os entrevistados apresentaram-se satisfeitos com o atendimento médico e de enfermagem, principalmente quanto ao respeito, privacidade, escuta e confidencialidade. Verificou-se que apesar dos profissionais de saúde se empenharem na prestação de um atendimento humanizado, foi evidente a fragmentação do cuidado na rede estudada, o que dificulta o seguimento adequado e oportuno ao paciente, refletindo negativamente na qualidade da assistência prestada.

Palavras-chave Atenção Primária à Saúde, Pesquisa sobre serviços de saúde, Satisfação do paciente 


\section{Introdução}

A Atenção Primária à Saúde (APS), enquanto porta de entrada preferencial do sistema de saúde brasileiro, deve atuar no processo de acolher o usuário promovendo a vinculação e corresponsabilização pelo seu cuidado. No entanto, muitos são os obstáculos para a prestação de uma assistência à saúde de qualidade, problemática esta que reflete negativamente sobre a continuidade do cuidado, integralidade e eficiência do siste$\mathrm{ma}^{1}$.

A importância que a APS tem para o sistema de saúde, assim como, a análise de seu impacto sobre a situação de saúde da população, requerem a realização de avaliações permanentes, com o objetivo de fortalecer seus resultados e a qualidade do cuidado prestado ${ }^{2,3}$.

Sob essa ótica, a qualidade da atenção prestada pelas Unidades de Saúde é considerada um imperativo técnico e social. A busca pela melhoria da equidade de acesso aos recursos, os altos custos da assistência, bem como os novos anseios da sociedade, tem direcionado os esforços das instituições, tanto particulares como públicas, no sentido de buscar evidências de que os serviços de saúde estão sendo providos eficientemente e que mantém e melhoram a qualidade do cuidado ao paciente ${ }^{4}$.

Considerando o conceito de qualidade desenvolvido por Donabedian ${ }^{5}$, a noção de satisfação do paciente tornou-se um dos elementos da avaliação dos serviços de saúde. Esse tipo de abordagem considera o paciente como sujeito ativo no processo avaliativo e coloca suas opiniões, expectativas e tudo o que ele considera justo ou equânime como elementos legítimos na definição e na mensuração da qualidade da atenção à saúde ${ }^{6}$.

Nesse contexto, a realização de estudos de satisfação dos usuários é considerada estratégica por permitir sua intervenção em planos e programas, com o intuito de influenciar na melhoria da assistência e subsidiar a construção de um novo aspecto do cuidado ${ }^{7,8}$.

Ademais, essa metodologia conquistou um importante papel no cenário brasileiro por impactar no fortalecimento da participação social na medida em que incorpora os usuários nos processos de planejamento e avaliação dos serviços de saúde 9 .

O direito à saúde precisa ser constantemente defendido e a avaliação apresenta-se como importante ferramenta. Avaliar a partir da experiência dos usuários possibilita ${ }^{10}$, não somente construir uma nova perspectiva de cuidado, como também monitorar a oferta dos serviços e fortalecer a participação social.

Nessa perspectiva, este estudo se propôs a analisar, a partir da percepção dos usuários, a qualidade da assistência prestada na APS em um município da região metropolitana do estado de Pernambuco.

\section{Métodos}

Trata-se de um estudo exploratório, de corte transversal e abordagem quantitativa, realizado em Unidades de Saúde da Família (USF) no município de Jaboatão dos Guararapes, localizado na Região Metropolitana do Recife.

No ano de 2018, o município possuía população de 697.636 habitantes, segundo estimativas do Instituto Brasileiro de Geografia e Estatística $(\mathrm{IBGE})^{11}$, estando entre os cinco principais do estado. Segundo o Ministério da Saúde, apresentava 104 equipes de Saúde da Família entre julho e setembro de 2018, período de coleta dos dados, representando uma cobertura de $51,43 \%$ da população ${ }^{12}$.

Para cálculo da amostra utilizou-se como referência o percentual de satisfação esperada segundo estudos de Gouveia ${ }^{13}$ que avaliou a satisfação dos usuários com a assistência à saúde no estado de Pernambuco.

Para definição das unidades a serem pesquisadas, considerou-se uma amostragem aleatória simples do tipo proporcional com um erro amostral de 5\%, erro relativo de 20\%, hipótese de $65 \%$ de satisfação e uma confiabilidade de $95 \%$, sendo assim calculado um tamanho amostral de 40 unidades de saúde que foram distribuídas aleatoriamente por meio de sorteio nas gerências regionais de saúde, considerando a proporção de cada regional com relação ao universo total de equipes. Em se tratando da amostra de usuários, considerou-se uma amostragem aleatória simples do tipo proporcional com um erro amostral de $5 \%$, efeito de desenho de 1,5 e uma confiabilidade de $95 \%$, sendo assim calculado um tamanho amostral de 525 usuários.

Os critérios para a inclusão dos usuários na pesquisa foram: a) indivíduos cadastrados e usuários da USF do município com idade igual ou superior a 18 anos que estivessem presentes no momento da entrevista e b) ter tido ao menos uma experiência de atendimento na USF nos últimos seis meses.

Para avaliação dos usuários sobre a qualidade da assistência prestada na APS definiu-se cinco 
dimensões: acessibilidade, estrutura, assistência clínica, relação profissional-usuário e atividades comunitárias. As dimensões foram definidas tomando- se como marco os referenciais da Avaliação do desempenho do Sistema de Saúde/Proadess $^{14}$, das políticas de qualificação ${ }^{15}$, assim como de Reid et al. ${ }^{16}$ e Beltran ${ }^{17}$. As questões relativas à satisfação apresentavam cinco alternativas para julgamento: péssimo, ruim, regular, bom e ótimo.

Foi aplicado um questionário estruturado, elaborado especificamente para os propósitos da pesquisa, constituído por 58 questões. A coleta de dados ocorreu na sala de espera das USF, durante todos os dias da semana e nos diferentes turnos de atendimento para garantir a aleatoriedade da participação.

A seleção dos indivíduos levou em consideração uma sistemática estabelecida de acordo com o local em que o usuário estava sentado à espera do atendimento. Todos os assentos foram previamente numerados e no momento da coleta, o entrevistador procedeu a realização de um sorteio aleatório simples para determinar quais assentos participariam da entrevista.

Para mensurar o grau de satisfação dos participantes, sob a hipótese de independência das observações, criou-se uma variável dicotômica, considerando que os atributos péssimo, ruim e regular referiam-se à insatisfação, e bom e ótimo, à satisfação.

Para verificar a existência de diferenças significativas entre as dimensões de satisfação e seus atributos optou-se pelo Teste de Friedman. Assim, foram apresentados os means rankings de satisfação entre os usuários, considerando quanto maior a média, maior a satisfação das participantes.

Cabe ressaltar que na avaliação de satisfação dos usuários pode haver o viés de gratidão, sentimento que pode ser aflorado devido à oferta pública do serviço bem como pelo receio de perder a garantia do atendimento em caso de avaliação negativa do serviço. A influência desse tipo de viés foi minimizada pelo esclarecimento por parte do entrevistador acerca dos objetivos da pesquisa e pela garantia do sigilo das informações fornecidas.

O estudo é parte dos resultados da tese de doutorado que teve o projeto aprovado pelo Comitê de Ética em Pesquisa do Instituto Aggeu Magalhães, da Fundação Oswaldo Cruz, no Recife, Pernambuco.

\section{Resultados}

Entre os 525 entrevistados que representam os usuários atendidos na APS em Jaboatão dos Guararapes, a maioria era do sexo feminino $(87,4 \%)$, negra/parda $(77,0 \%)$, com idade entre 20 e 49 anos $(61,7 \%)$, com renda de até 01 salário mínimo $(64,2 \%)$, casados ou em união estável $(67,0$ $\%$ ) e com filhos (média $=2,4$ filhos).

Quanto à escolaridade, destacaram-se os pacientes que cursaram o ensino fundamental de forma incompleta $(38,2 \%)$ e os de ensino médio completo (35,3\%). Em relação à ocupação, a maioria se declarou Do Lar $(27,4 \%)$, seguido dos desempregados (26,9\%) e empregados/autônomos que representaram $24,4 \%$ dos entrevistados.

A análise da satisfação dos usuários apresentou diferenças significativas $(p=0,00)$ entre as dimensões avaliadas, sendo que a acessibilidade e a estrutura apresentaram os maiores valores de insatisfação. Por outro lado, a relação profissional - usuário apresentou a maior proporção de usuários satisfeitos $(81,8 \%)$ (Tabela 1$)$.

$\mathrm{Na}$ avaliação da acessibilidade, identificou-se a maior prevalência de insatisfação dos usuários relacionados ao acesso à consulta $(88,8 \%)$ e exames $(90,8 \%)$ especializados, bem como ao tempo de espera para atendimento na própria unidade $(69,0 \%)$. As categorias que apresentaram maior nível de satisfação foram a distância percorrida até o posto $(67,3 \%)$ e o horário de atendimento dos profissionais $(65,5 \%)$ (Tabela 2$)$.

Ainda na Tabela 2, observou-se que a dimensão estrutura apresentou valores maiores que $60 \%$ de insatisfação dos usuários na maioria das categorias avaliadas. Os aspectos que geraram maior nível de insatisfação dizem respeito à quantidade/conforto das cadeiras (65,5\% e $67,9 \%)$, ventilação/temperatura $(63 \%)$ e a estrutura física da unidade $(62,9 \%)$. Por outro lado, as categorias limpeza da unidade e limpeza do banheiro contaram com $40,4 \%$ e $45,0 \%$ de insatisfeitos, respectivamente.

As categorias relacionadas à assistência clínica apresentaram um nível de satisfação maior que $70 \%$, com exceção apenas para a categoria atendimento do Agente Comunitário de Saúde (ACS) que apresentou $68,2 \%$ de usuários satisfeitos. Do mesmo modo, a maioria das categorias da relação profissional-usuário alcançaram valores maiores que $70 \%$ de satisfação com destaque para as categorias: tratamento respeitoso por parte de médi$\cos (93,5 \%)$ e enfermeiros $(95,8 \%)$, e a confiança atribuída aos enfermeiros (90,7\%). Entretanto, a possibilidade de realizar reclamações e a presteza 
no atendimento apresentaram os menores valores de satisfação (Tabela 3).

$\mathrm{Na}$ Tabela 4, identificou-se que a dimensão desenvolvimento de atividades comunitárias apresentou valores de satisfação menores que 70\% dentre os entrevistados, com exceção apenas para a avaliação das atividades em grupo (72,0\%). As categorias diretamente relacionadas à atuação dos ACS apresentaram valores de insatisfação, variando entre $44,9 \%$ e $55,7 \%$. Dentre os entrevistados que tiveram experiência com visita domiciliar pelo médico e enfermeiro, mais de $60 \%$ se mostraram satisfeitos com esse tipo de atendimento.

\section{Discussão}

A avaliação da assistência à saúde sob a perspectiva do usuário é uma ferramenta de grande importância para identificar aspectos e situações que interferem positivamente no atendimento à população, bem como identificar os principais fatores geradores de insatisfação. O olhar do usuário representa o melhor instrumento de avaliação e participação social na busca por um sistema de saúde público de qualidade que se pretende equânime e universal, com vistas a atender as reais necessidades da sua população.

A maioria dos usuários da APS são mulheres jovens (entre 20 e 39 anos de idade), que se consideram negras ou pardas, com baixo nível de

Tabela 1. Dimensões da qualidade da satisfação dos usuários com os cuidados na Atenção Primária à Saúde de Jaboatão dos Guararapes, Pernambuco, Brasil, 2018.

\begin{tabular}{|c|c|c|c|c|c|c|c|}
\hline \multirow{2}{*}{ Dimensões } & \multirow{2}{*}{$\begin{array}{c}\text { Mean } \\
\text { Ranking }\end{array}$} & \multirow{2}{*}{${ }^{\star}$ Respostas } & \multicolumn{2}{|c|}{ Insatisfeito } & \multicolumn{2}{|c|}{ Satisfeito } & \multirow[t]{2}{*}{$\mathrm{X}^{2}$} \\
\hline & & & $\mathbf{n}$ & $\%$ & $\mathbf{n}$ & $\%$ & \\
\hline Acessibilidade & 3,39 & 488 & 293 & 60,0 & 195 & 40,0 & \multirow{5}{*}{$\begin{array}{c}X^{2}=483,7 \\
D F=4 \\
p< \\
0,00001\end{array}$} \\
\hline Assistência Clínica & 2,41 & 483 & 105 & 21,7 & 378 & 78,3 & \\
\hline Relação Profissional-Usuário & 2,41 & 463 & 84 & 18,2 & 378 & 81,8 & \\
\hline Desenvolvimento de Atividades Comunitárias & 3,49 & 321 & 139 & 43,3 & 182 & 56,7 & \\
\hline Estrutura & 3,30 & 498 & 292 & 58,7 & 206 & 41,3 & \\
\hline
\end{tabular}

Fonte: Trabalho de campo realizado no município de Jaboatão dos Guararapes no período de junho a dezembro de 2018.

Tabela 2. Satisfação dos usuários com a Acessibilidade e a Estrutura da Atenção Primária à Saúde de Jaboatão dos Guararapes, Pernambuco, Brasil, 2018.

\begin{tabular}{|c|c|c|c|c|c|c|c|}
\hline \multirow{2}{*}{ Acessibilidade } & \multirow{2}{*}{$\begin{array}{c}\text { Mean } \\
\text { Ranking }\end{array}$} & \multirow{2}{*}{${ }^{\star}$ Respostas } & \multicolumn{2}{|c|}{ Insatisfeito } & \multicolumn{2}{|c|}{ Satisfeito } & \multirow[b]{2}{*}{$\mathrm{X}^{2}$} \\
\hline & & & $\mathbf{n}$ & $\%$ & $\mathbf{n}$ & $\%$ & \\
\hline Distância percorrida até o posto & 3,38 & 525 & 172 & 32,8 & 353 & 67,2 & \multirow{8}{*}{$\begin{array}{c}\mathrm{X}^{2}=516,5 \\
\mathrm{DF}=7 \\
\mathrm{p}< \\
0,00001\end{array}$} \\
\hline Facilidade de agendamento de consultas & 4,47 & 523 & 314 & 60,0 & 209 & 40,0 & \\
\hline Horário de atendimento dos profissionais & 3,47 & 521 & 180 & 34,5 & 341 & 65,5 & \\
\hline Tempo de espera para atendimento & 4,79 & 519 & 358 & 69,0 & 161 & 31,0 & \\
\hline Acesso no agravamento do estado de saúde & 4,28 & 484 & 272 & 56,2 & 212 & 43,8 & \\
\hline Disponibilidade de medicamentos & 4,45 & 496 & 296 & 59,7 & 200 & 40,3 & \\
\hline Acesso à consulta especializada & 5,56 & 421 & 374 & 88,8 & 47 & 11,2 & \\
\hline Acesso a exames especializados & 5,6 & 413 & 375 & 90,8 & 38 & 9,2 & \\
\hline \multicolumn{8}{|l|}{ Estrutura } \\
\hline Estrutura física da unidade & 4,12 & 525 & 330 & 62,9 & 195 & 37,1 & \multirow{7}{*}{$\begin{array}{c}\mathrm{X}^{2}=145,1 \\
\mathrm{DF}=6 \\
\mathrm{p}< \\
0,00001\end{array}$} \\
\hline Quantidade de cadeiras & 4,27 & 522 & 342 & 65,5 & 180 & 34,5 & \\
\hline Conforto das cadeiras & 4,34 & 523 & 355 & 67,9 & 168 & 32,1 & \\
\hline Conservação dos equipamentos & 4,08 & 499 & 309 & 61,9 & 190 & 38,1 & \\
\hline Limpeza da unidade & 3,42 & 522 & 211 & 40,4 & 311 & 59,6 & \\
\hline Limpeza do banheiro & 3,55 & 369 & 166 & 45,0 & 203 & 55,0 & \\
\hline Ventilação e temperatura & 4,25 & 524 & 330 & 63,0 & 194 & 37,0 & \\
\hline
\end{tabular}

Fonte: Trabalho de campo realizado no município de Jaboatão dos Guararapes no período de junho a dezembro de 2018. 
Tabela 3. Satisfação dos usuários com a Assistência clínica e a Relação profissional-usuário da Atenção Primária à Saúde de Jaboatão dos Guararapes, Pernambuco, Brasil, 2018.

\begin{tabular}{|c|c|c|c|c|c|c|c|}
\hline \multirow{2}{*}{ Assistência Clínica } & \multirow{2}{*}{$\begin{array}{c}\text { Mean } \\
\text { Ranking }\end{array}$} & \multirow{2}{*}{${ }^{\star}$ Respostas } & \multicolumn{2}{|c|}{ Insatisfeito } & \multicolumn{2}{|c|}{ Satisfeito } & \multirow[b]{2}{*}{$\mathbf{X}^{2}$} \\
\hline & & & n & $\%$ & n & $\%$ & \\
\hline Atendimento médico & 4,3 & 477 & 66 & 13,8 & 411 & 86,2 & \multirow{8}{*}{$\begin{array}{c}\mathrm{X}^{2}=96,3 \\
\mathrm{DF}=7 \\
\mathrm{p}< \\
0,00001\end{array}$} \\
\hline Atendimento de enfermagem & 4,06 & 434 & 44 & 10,1 & 390 & 89,9 & \\
\hline Atendimento do técnico de enfermagem & 4,15 & 452 & 65 & 14,4 & 387 & 85,6 & \\
\hline Atendimento do ACS & 4,66 & 494 & 157 & 31,8 & 337 & 68,2 & \\
\hline Realização de procedimentos & 4,73 & 452 & 132 & 29,2 & 320 & 70,8 & \\
\hline Exame clínico & 4,57 & 517 & 107 & 20,7 & 410 & 79,3 & \\
\hline Duração da consulta & 4,73 & 522 & 127 & 24,3 & 395 & 75,7 & \\
\hline Resolução de suas necessidades & 4,8 & 515 & 139 & 27,0 & 376 & 73,0 & \\
\hline \multicolumn{8}{|l|}{ Relação Profissional-Usuário } \\
\hline Tratamento respeitoso pelo (a) médico (a) & 4,82 & 475 & 31 & 6,5 & 444 & 93,5 & \multirow{10}{*}{$\begin{array}{c}\mathrm{X}^{2}= \\
504,7 \\
\mathrm{DF}=9 \\
\mathrm{p}< \\
0,00001\end{array}$} \\
\hline Tratamento respeitoso pelo (a) enfermeiro (a) & 4,45 & 428 & 18 & 4,2 & 410 & 95,8 & \\
\hline Escuta do profissional & 5,83 & 518 & 110 & 21,2 & 408 & 78,8 & \\
\hline Confiança no (a) médico (a) & 5,27 & 470 & 65 & 13,8 & 405 & 86,2 & \\
\hline Confiança no (a) enfermeiro (a) & 4,75 & 421 & 39 & 9,3 & 382 & 90,7 & \\
\hline Privacidade & 5,33 & 514 & 76 & 14,8 & 438 & 85,2 & \\
\hline Informações prestadas com clareza & 5,24 & 525 & 69 & 13,1 & 456 & 86,9 & \\
\hline Confidencialidade das informações & 5,15 & 505 & 58 & 11,5 & 447 & 88,5 & \\
\hline Possibilidade de realizar reclamações & 7,72 & 294 & 216 & 73,5 & 78 & 26,5 & \\
\hline Presteza no atendimento & 6,44 & 477 & 162 & 34,0 & 315 & 66,0 & \\
\hline
\end{tabular}

Fonte: Trabalho de campo realizado no município de Jaboatão dos Guararapes no período de junho a dezembro de 2018.

Tabela 4. Satisfação dos usuários com o Desenvolvimento de atividades comunitárias da Atenção Primária à Saúde de Jaboatão dos Guararapes, Pernambuco, Brasil, 2018.

\begin{tabular}{|c|c|c|c|c|c|c|c|}
\hline \multirow{2}{*}{ Desenvolvimento de Atividades Comunitárias } & \multirow{2}{*}{$\begin{array}{c}\text { Mean } \\
\text { Ranking }\end{array}$} & \multirow{2}{*}{${ }^{\star}$ Respostas } & \multicolumn{2}{|c|}{ Insatisfeito } & \multicolumn{2}{|c|}{ Satisfeito } & \multirow[t]{2}{*}{$\mathrm{X}^{2}$} \\
\hline & & & $\mathbf{n}$ & $\%$ & $\mathbf{n}$ & $\%$ & \\
\hline Palestras & 3,79 & 303 & 95 & 31,4 & 208 & 68,6 & \\
\hline Atividades em grupos & 3,6 & 192 & 52 & 27,1 & 140 & 72,9 & \\
\hline Visita domiciliar do ACS & 4,17 & 492 & 274 & 55,7 & 218 & 44,3 & $\mathrm{X}^{2}=10,4$ \\
\hline Orientações prestadas pelo ACS & 4,05 & 492 & 221 & 44,9 & 271 & 55,1 & $\mathrm{DF}=6$ \\
\hline Acompanhamento do estado de saúde pelo ACS & 4,05 & 488 & 232 & 47,5 & 256 & 52,5 & $\mathrm{p}=0,111$ \\
\hline Visita domiciliar pelo(a) médico(a)* & 4,24 & 136 & 47 & 34,6 & 89 & 65,4 & \\
\hline Visita domiciliar pelo $(a)$ enfermeiro $(a)^{*}$ & 4,11 & 142 & 51 & 35,9 & 91 & 64,1 & \\
\hline
\end{tabular}

${ }^{\star}$ A visita domiciliar é direcionada a grupos específicos (acamados, puérperas, usuários com deficiência, etc) e portanto, os resultados obtidos representam os usuários que tiveram experiência com esse tipo de atendimento.

Fonte: Trabalho de campo realizado no município de Jaboatão dos Guararapes no período de junho a dezembro de 2018.

escolaridade e com filhos. A maioria é casada e se considera Do Lar ou desempregada, assim como encontrado em outros estudos ${ }^{18,19}$.

A maior procura pelo atendimento por mulheres pode ser explicada pelo fato de elas se preocuparem mais com o estado de saúde e também por serem aquelas que geralmente acompanham filhos e esposos para atendimento. Em detrimen- to disso, os homens parecem dedicar-se mais às atividades laborais e consequentemente deixam as questões de saúde em segundo plano.

Percebeu-se uma maior utilização dos serviços por indivíduos que estão fora do mercado de trabalho. Os usuários que se identificaram como desempregados, Do Lar e aposentado/pensionista/ em beneficio representam mais de $70 \%$ do total 
de pessoas que foram abordados mediante a utilização do serviço realizado pelas USF do município em estudo. Esses dados apontam para a maior dificuldade de acesso por parte da população economicamente ativa.

O maior desafio nesse caso é a dificuldade em desenvolver estratégias de promoção à saúde e prevenção de doenças para esse grupo. Como consequência, aumentam os casos em que o diagnóstico é tardio, implicando em complicações que requerem um manejo mais complexo e que na maioria das vezes não pode ser resolvido na APS.

Nesse contexto, esforços têm sido dirigidos para ampliar a oferta de serviços do SUS através do horário estendido de funcionamento das USF, contemplando o turno noturno, a fim de favorecer a participação dessa classe no cuidado à saúde ${ }^{20}$.

Entre as dimensões estudadas, a assistência clínica e a relação profissional-usuário foram as que apresentaram melhor avaliação pelos usuários, apresentando mais de $70 \%$ de aprovação. Por outro lado, a acessibilidade, a estrutura fisica e o desenvolvimento de atividades comunitárias contaram com mais de $40 \%$ de insatisfação.

Em relação à acessibilidade, nenhuma das categorias avaliadas foi considerada como satisfatória. A insatisfação recaiu principalmente sobre o acesso a consultas e exames especializados, o que retrata fragilidade no acesso à atenção especializada. Na maioria das vezes, esse fato deve-se a uma falta de organização dos serviços de acordo com a oferta e demanda, representada por uma capacidade de atendimento nas unidades especializadas e número de profissionais aquém da necessidade real da população. Como consequência disso, há longas filas de espera que comprometem o seguimento adequado do paciente e dificultam o tratamento oportuno e a resolubilidade dos casos.

Observou-se que, embora a distância percorrida até o posto de saúde e o horário de atendimento dos profissionais tenham alcançado cerca de $60 \%$ de aprovação, o atendimento na própria unidade foi motivo de grande insatisfação no que tange à facilidade de agendar consultas, acesso no agravamento de saúde, tempo de espera para atendimento e disponibilidade de medicamentos.

A distância entre a casa do usuário e o posto de saúde é um importante item associado à satisfação dos usuários. No presente estudo, mais de $30 \%$ dos entrevistados estiveram insatisfeitos com essa variável. A localização da USF deve ser planejada considerando os aspectos geográficos de cada território a fim de facilitar o acesso da população adstrita. Na prática, muitas vezes essa questão é negligenciada pelos gestores e as unidades de saúde estão inseridas em locais de difícil acesso. Também é comum identificar a utilização de uma mesma estrutura para comportar várias equipes, o que favorece o distanciamento de algumas áreas, dificultando o deslocamento dos pacientes que por vezes, necessitam utilizar transporte público para chegar à unidade.

Estudo realizado no Brasil ${ }^{21}$ identificou que se a distância da residência do usuário até a unidade de saúde é considerada moderada ou distante, a chance de satisfação sofre uma redução de $32 \%$ e $16 \%$, respectivamente. Para Santos et al. ${ }^{8}$ o fácil acesso geográfico é importante para a procura pelos serviços de saúde, influenciando na busca pelo atendimento, acompanhamento e tratamento.

A facilidade em agendar consultas, $o$ acesso no agravamento de saúde e o tempo de espera para atendimento foram apontados como insatisfatórios por mais de $50 \%$ dos entrevistados. Considerando que as USF devem atender a uma população residente em território delimitado e sob a responsabilidade sanitária da equipe de saúde, é preciso repensar o planejamento das ações e serviços necessários para atender as singularidades desse território.

Estudo realizado em uma Clínica Escola ${ }^{22}$ identificou a dificuldade de marcação da primeira consulta como a principal reclamação mencionada pelos pacientes. Quanto ao fato de não conseguir atendimento sem agendamento prévio, Protassio et al. ${ }^{23}$ verificaram que essa situação diminui a chance de satisfação do usuário em $42 \%$.

$\mathrm{O}$ tempo de espera para atendimento é fator reconhecidamente relacionado com a insatisfação dos usuários ${ }^{18,23,24,25}$. Nesse estudo, identificou-se um tempo médio de espera para atendimento bastante variável entre as equipes estudadas, sendo mais prevalente o período de espera de até sete dias para conseguir a consulta na USF. Entretanto, destaca-se que 17\% dos entrevistados conseguiram atendimento no mesmo dia, revelando que algumas equipes são sensíveis ao acolhimento adequado e oportuno da população de forma a reconhecer os casos que necessitam de atendimento prioritário.

Em relação à disponibilidade de medicamentos, cerca de $60 \%$ de usuários estavam insatisfeitos com a dispensação de medicamentos, o que pode estar relacionado a falhas no processo de planejamento e distribuição desses insumos, resultando na falta de remédios e interferência no 
tratamento em casos agudos e crônicos. Fato que se reveste de importância, uma vez que esse estudo identificou que a maioria dos usuários da APS estão inseridos em famílias em situação de vulnerabilidade.

Corrobora com esses achados, o estudo de Gabe et al. ${ }^{22}$ que verificou elevado nível de insatisfação dos usuários quanto ao acesso a medicamentos em uma clínica escola. Por outro lado, Soeiro et al. ${ }^{26}$ identificou $58,4 \%$ de usuários satisfeitos com esses serviços.

A avaliação da estrutura física por sua vez, apresentou um alto nível de insatisfação da população estudada, corroborando estudos anteriores ${ }^{8,19,27}$. As categorias alcançaram cerca de $60 \%$ de desaprovação com exceção apenas para os aspectos relacionados à limpeza do ambiente.

A insatisfação dos usuários com a estrutura física pode interferir negativamente nos processos de acolher e atender as demandas da comunidade. Para Lucena et al. ${ }^{19}$, a promoção de um ambiente de qualidade estimula o processo de reflexão das práticas e dos modos de operar naquele território, construindo locais produtores de saúde, de forma que além de funcionais, proporcionam espaços vivenciais prazerosos.

Os espaços devem ser adequados ao contexto em que a unidade está inserida, levando em consideração que o território vivo e singular, é representado pelas peculiaridades da população cadastrada, número de usuários esperados e serviços ofertados, de forma a garantir um atendimento humanizado e acolhedor.

Em relação à dimensão assistência clínica, verificou-se um alto nível de satisfação, contemplando mais de $70 \%$ de aprovação para todas as categorias estudadas, exceto para o atendimento do ACS.

$\mathrm{O}$ atendimento dos profissionais que contemplam as categorias médica e de enfermagem foi responsável pelo mais alto grau de satisfação dessa dimensão. Além disso, os aspectos relacionados ao exame clínico, duração da consulta, realização de procedimentos e resolução das suas necessidades foram muito bem avaliados pelos entrevistados. Isso aponta para a prestação de cuidados que preza pelo atendimento humanizado e favorece o vínculo da população com a equipe, repercutindo em maiores chances de sucesso na promoção, prevenção e tratamento dos problemas de saúde dos usuários.

Estudos realizado no Brasil e Espanha identificaram um percentual de mais de $80 \%$ dos usuários satisfeitos com a atenção da equipe que trabalha nas unidades de atenção primária ${ }^{7,28}$.
No que tange à resolução das necessidades dos usuários, os achados sugerem um maior comprometimento dos profissionais da APS em buscar respostas efetivas para os problemas de saúde dos usuários do território. A integralidade da assistência está diretamente relacionada à capacidade resolutiva da equipe. Estudo realizado no Brasil ${ }^{21}$ identificou que o fato do usuário resolver seus problemas de saúde na própria unidade foi um dos fatores mais importantes para contemplar a sua satisfação em todas as regiões do território nacional.

A dimensão relação profissional-usuário apresentou valores elevados de satisfação na maioria das categorias avaliadas, destacando-se o tratamento baseado no respeito e confiança por parte dos médicos e enfermeiros, a privacidade preservada e a confidencialidade das informações. $\mathrm{O}$ atendimento pautado no respeito, confiança, privacidade e confidencialidade são propícios ao desenvolvimento de vínculo do profissional com o paciente. Isso favorece a busca pelos serviços de saúde por parte do usuário e impacta positivamente no seguimento adequado dos casos.

Rodrigues et al. ${ }^{29}$ identificaram a satisfação relacionada à confiança nos profissionais em mais de $80 \%$ dos usuários atendidos na APS. Estudos realizados com gestantes e idosos ${ }^{25,27}$, identificaram elevado percentual de satisfação de usuários que consideraram o tratamento como respeitoso por parte dos profissionais de saúde.

Corroborando com os achados desse estudo, outros autores ${ }^{25,30,31}$ apontam a boa avaliação por parte dos entrevistados quanto às categorias privacidade e confidencialidade. Por outro lado, existem relatos que identificaram serviços onde a privacidade nas consultas foi identificada pelos usuários como uma das maiores falhas no atendimento prestado ${ }^{27}$.

Cabe destacar que aspectos relacionados a uma comunicação adequada durante a consulta foram muito bem avaliadas nos quesitos escuta e clareza das informações prestadas, em conformidade com a literatura ${ }^{19,25,29}$. A comunicação adequada é um importante fator a ser considerado para prestação de um atendimento de qualidade principalmente no que concerne à relação de diálogo com o paciente com vistas à promoção de uma escuta adequada, bem como a oferta de explicações claras a respeito do quadro de saúde e/ou tratamento.

A possibilidade de realizar reclamações apresentou baixos níveis de satisfação ao ser avaliada pelos entrevistados, realidade que vem sendo amplamente discutida na literatura nacional e 
internacional ${ }^{21,27-29}$. Isso gera preocupação, uma vez que os usuários não encontram abertura para expor as suas insatisfações, ou quando a queixa é registrada, muitas vezes não é resolvida, tornando negativa a percepção dos pacientes. Esse fato sugere a falta de comprometimento ético dos profissionais, representado pelo baixo interesse em solucionar as reclamações e reivindicações da população adstrita.

Quanto ao desenvolvimento de atividades comunitárias, apenas a categoria atividades em grupo alcançou avaliação satisfatória. Mais de $40 \%$ dos entrevistados apresentarem-se insatisfeitos com a atuação dos ACS no que concerne às visitas, orientações e acompanhamento do estado de saúde.

O ACS é reconhecidamente um importante ator no desenvolvimento de ações comunitárias. Ele convive com situações semelhantes aos usuários do serviço, o que possibilita criar uma relação de aproximação com os moradores e compreender a realidade local ${ }^{32,33}$.

No entanto, os achados desse estudo sugerem que apenas o vínculo estabelecido com a comunidade não é suficiente para alcançar a satisfação por parte dos usuários. Embora a visita domiciliar perpasse a questão da escuta e do acolhimento, deixa a desejar na frequência com que é realizada e nas orientações fornecidas.

No que se refere às visitas domiciliares por médicos e enfermeiros, boa parte dos entrevistados mostrou-se satisfeito, embora cerca de 30\% desaprovaram esse tipo de atividade. Esse fato também representa uma fragilidade no que diz respeito ao acesso ao serviço de saúde considerando os grupos que apresentam dificuldade de locomoção. É importante repensar o processo de trabalho dos profissionais a fim de garantir o acesso universal ao serviço.

A visita domiciliar é um importante instrumento para o conhecimento dos determinantes e condicionantes do processo saúde-doença. Por meio dessa ação, é possível conhecer o contexto de vida na qual as famílias estão inseridas, seus aspectos sociais, ambientais, habitacionais, etc. Esse entendimento favorece o planejamento e execução de estratégias assistenciais individualizadas de acordo com a realidade de cada caso.

Faz-se necessário o investimento e desenvolvimento de atividades comunitárias com o intuito de romper o modelo centrado na doença e avançar nos aspectos que envolvem a promoção à saúde.
Para tanto, devem ser promovidas atividades com a participação de toda a equipe de saúde, priorizando o vínculo mais estreito com a comunidade, a inserção das visitas domiciliares como rotina do processo de trabalho e a realização de atividades educativas que forneçam subsídios ao autocuidado e fortaleçam a autonomia dos usuários.

\section{Considerações finais}

Considerar o lugar de fala dos sujeitos envolvidos com o cuidado à saúde é fundamental para assegurar um direito que tem sido fortemente ameaçado. Esse espaço apresenta-se ainda mais importante quando retrata em sua tela, a vulnerabilidade que caracteriza nossa sociedade. A avaliação apresentada identificou que a maioria era composta por mulheres jovens, com filhos, que se consideram negras ou pardas, com baixo nível de escolaridade e renda.

No contexto de desigualdade, importantes aspectos estavam marcados pela insatisfação. As dimensões de acessibilidade, desenvolvimento de atividades comunitárias e estrutura física foram aquelas pior avaliadas pelos participantes do estudo. Dimensões essenciais ao processo de acolhimento e fortalecimento do vínculo com a comunidade no território bem como, à oferta de uma assistência de qualidade, capaz de promover um atendimento integral e, resolutivo.

Por outro lado, os usuários apresentaram-se satisfeitos quanto à assistência clínica e o relacionamento com os profissionais de saúde. Isso demonstra o compromisso dos profissionais em buscar um atendimento humanizado e de qualidade que preza pelo vínculo, respeito e confiança, mesmo diante das dificuldades estruturais e de acesso a medicamentos, exames e consultas especializadas.

A realização de estudos que investiguem e reflitam sobre a oferta de ações e serviços de saúde é essencial para assegurar que o compromisso com o direito à saúde seja resguardado. $\mathrm{O}$ investimento na APS enquanto ordenadora do cuidado é essencial. Para tanto, conhecer as perspectivas de usuários pode ser considerado um importante subsídio ao planejamento de estratégias efetivas por parte dos gestores a fim de defender no território, o respeito às subjetividades, a dignidade, a integralidade da atenção e a garantia de uma assistência resolutiva e de qualidade. 


\section{Colaboradores}

DS Melo participou da concepção, delineamento, análise e interpretação dos dados e redação do artigo. ALA Silva participou da análise e interpretação dos dados. PJL Martelli participou da revisão crítica. TM Lyra participou da revisão crítica. GMD Miranda participou da análise e interpretação dos dados e redação do artigo. ACG Mendes participou do delineamento, análise e interpretação dos dados e aprovação da versão a ser publicada.

\section{Referências}

1. Bousquat A, Giovanella L, Campos EMS, Almeida PF, Martins CL, Mota PHS, Mendoça MHM de, Medina MG, Viana ALA, Fausto MCR, Paula DB. Atenção primária à saúde e coordenação do cuidado nas regiões de saúde: perspectiva de gestores e usuários. Cienc Saude Colet 2017; 22(4):1141-1154.

2. Campos RTO, Ferrer AL, Gama CAP, Campos GWS, Trapé TL, Dantas DV. Avaliação da qualidade do acesso na atenção primária de uma grande cidade brasileira na perspectiva dos usuários. Saude Debate 2014; 38(n. spe.):252-264.

3. Carneiro MSM, Melo DMS, Gomes JM, Pinto, FJM, Silva MGC. Avaliação do atributo coordenação da atenção primária à saúde: aplicação do PCATool a profissionais e usuários. Saude Debate 2014; 38(n. spe.).

4. Nery NG, Marcelo VC, Dantas MAA. A satisfação de idosos quanto à Estratégia Saúde da Família, a partir da atenção em Saúde Bucal. Rev Brasil Cien Saude 2010; 14 (1):43-50.

5. Donabedian A. La calidad de la atención médica definición y métodos de evaluación. Prensa Médica Mexicana 1984.

6. Almeida PF, Medina MG, Fausto MCR, Giovanella L, Bousquat A, Mendonça MHM. Coordenação do cuidado e Atenção Primária à Saúde no Sistema Único de Saúde. Saude Debate 2018; 42(spe. 1):244-260.

7. Callou Filho CR, Machado MFAS, Mesquita CAM, Rocha LK, Rodrigues MVS. Estratégia Saúde da Família: satisfação dos usuários no município de FortalezaCE. Rev Saude Pesq 2017; 10(2):339-346.

8. Santos AL, Andrade CJN, Santos MS, Silva Neto MF, Porto TD. Percepção de usuários com diabetes acerca da assistência recebida na Atenção Primária à Saúde. Rev APS 2018; 21(2): 251-258.

9. Esperidião MA, Viera da Silva LM. The satisfaction of the user in health services evaluation: essay on the imposition of problems. Saude Debate 2018; 42(2):331340.

10. Brandão ALRBS, Giovanella L, Campos CEA. Avaliação da atenção básica pela perspectiva dos usuários: adaptação do instrumento EUROPEP para grandes centros urbanos brasileiros. Cien Saude Colet 2013; 18(1):103-114.

11. Brasil. Departamento de Informática do SUS. População residente - estimativas para o TCU - Pernambuco. [acessado 2020 ago 18]. Disponível em: http://tabnet. datasus.gov.br/cgi/tabcgi.exe?ibge/cnv/poptpe.def.

12. Brasil. Cobertura da Atenção Básica. [acessado 2020 ago 18]. Disponível em: https://egestorab.saude.gov. br/paginas/acessoPublico/relatorios/relHistoricoCoberturaAB.xhtml;jsessionid $=6 \mathrm{NmUeRNj6g6r8JTL}$ p5WRvZAh.

13. Gouveia GC, Souza WV, Luna CF, Szwarcwald CL, Souza Júnior PRB. Satisfação dos usuários com a assistência de saúde no estado de Pernambuco, Brasil, 2005 Cienc Saude Colet 2011; 16(3):1849-1862.

14. Fundação Oswaldo Cruz (Fiocruz). Projeto - Desenvolvimento de metodologia de avaliação do desempenho do sistema de saúde Brasileiro/ PRO-ADESS. Relatório Final. Rio de Janeiro: Fiocruz; 2003. 
15. Brasil. Ministério da Saúde (MS). Secretaria de Atenção a Saúde. Departamento de Atenção Básica. Política Nacional da Atenção Básica. Brasília: MS; 2012.

16. Reid R, Haggerty J, Mckendry R. Defusing the confusion: concepts and measures of continuity of healthcare. Ottawa: Canadian Health Services Research Foundation; 2002. [cited 2020 fev 24]. Available from: http:// www.cfhi-fcass.ca/Migrated/PDF/ResearchReports/ CommissionedResearch/cr_contcare_e.pdf

17. Beltran TP. Coordinación entre Niveles Asistenciales. Una Propuesta para su Evaluación. Universitat Pompeu Fabra (UPF). Máster de Salud Pública; 2005-2006.

18. Abreu DMX, Araújo LHL, Reis CMR, Lima AMLD, Santos AF, Jorge AO, Fonseca Sobrinho D, Machado ATGM. Percepção dos usuários sobre o cuidado prestado por equipes participantes do Programa Nacional de Melhoria do Acesso e da Qualidade da Atenção Básica no Brasil. Epidemiol Serv Saude [periódico na Internet]. 2018 [acessado 2020 jan. 12]; 27(3): e00184715. [cerca de 13p.] Disponível em: https://doi.org/10.5123/S1679-49742018000300002.

19. Lucena LN, Melo RHV, Rodrigues MP, Andrade FB, Vilar RLA, Bosco Filho J. Avaliação da satisfação do usuário com o acolhimento na Estratégia da Saúde da Família no Recife (PE). Rev Cien Plural 2018; 4(2):2137.

20. Brasil. Ministério da Saúde(MS). Gabinete do Ministro. Portaria no 930, de 15 de maio de 2019. Institui o Programa Saúde na Hora. Brasília: MS; 2019.

21. Protasio APL, Gomes LB, Machado LS, Valenca AMG. Satisfação do usuário da Atenção Básica em Saúde por regiões do Brasil: 1o ciclo de avaliação externa do PMAQ-AB. Cien Saude Colet 2017; 22(6):1829-1844.

22. Gabe MC, Rosa AMS, Garcia LSB, Ambrosio PG, Madeira K. Avaliação do grau de satisfação de pacientes atendidos no serviço de medicina de uma clínica escola. Arq Catarin Med 2018; 47(3):146-158.

23. Protasio APL, Gomes LB, Machado LS, Valenca AMG. Factors associated with user satisfaction regarding treatment offered in Brazilian primary health care. Cad Saude Publica [periódico na Internet]. 2017 Mar. [acessado 2020 jan. 12]; 33(2): e00184715. [cerca de 13p.]. Disponível em: doi: 10.1590/0102-311 X00184715.

24. La Torre Mantilla AR, Oyola García AE, Quispe Ilanzo MP. Factores associados al grado de satisfacción del usuario del consultório externo de gastroenterología del Hospital Central de la Fuerza Aérea del Perú. Mayo, 2014. Rev Gerenc Polit Salud 2018; 17(34):1-10.

25. Melo DS, Martins RD, Jesus RPFS, Samico IC, Santo ACGE do. Avaliação da responsividade de um serviço de saúde público sob a perspectiva do usuário idoso. Rev Saude Publica [periódico na Internet]. 2017 [acessado 2020 jan. 12]; 51(62): [cerca de 13p.]. Disponível em: https://doi.org/10.1590/S15188787.2017051006854.
26. Soeiro OM, Tavares NUL, Nascimento Júnior JM, Guerra Junior AA, Costa EA, Acurcio FA, Guibu IA, Juliana Álvares J, Oliveira MGO Karnikowski, Leite SN, Costa KS. Satisfação dos usuários com os serviços da assistência farmacêutica na atenção básica nos municípios brasileiros. Rev Saude Publica 2017; 51(Supl. 2):21s.

27. Silva SN, Lima MG, Ruas CM. Avaliação de Serviços de Saúde Mental Brasileiros: satisfação dos usuários e fatores associados. Cien Saude Colet 2018; 23(11):3799-3810.

28. Juan Fariño C, Elsa VLF, Gabriela CMA, Paola VDA, Jesus LNM, Guadalupe SJD. Satisfacción de usuarios y calidad de atención en unidades primarias de Salud de Milagro. INSPILIP 2018; 2(2):2-25.

29. Rodrigues KZ, Mattos CFP, Ferreira DA, Koch LFA, Schmitt EJ, Gabardo MCL. Grau de satisfação entre os usuários de uma unidade básica de saúde no estado do Paraná, Brasil. Sci Med [periódico na Internet]. 2018 [acessado 2020 jan 12]; 28(4): [cerca de 13p.]. Disponível em:10.15448/1980-6108.2018.4.32253.

30. Hermida PMV, Nascimento ERP, Echevarría-Guanilo ME, Vituri DW, Martins SR, Barbosa SS. Responsividade do acolhimento com clasificação de risco: avaliação dos usuários em unidade de pronto atendimento. Texto Contexto Enferm [periódico na Internet]. 2019 [acessado 2020 jan 12]; 28: e20180480 [cerca de 10p.]. Disponível em: https://doi.org/10.1590/1980-265XTCE-2017-0480.

31. Dantas RAN, Dantas DV, Nascimento JCP, Sarmento SDG, Oliveira SP. Evaluation of satisfaction of users assisted by prehospital mobile emergency care servisse. Saude Debate 2018; 42(116):191-202.

32. Andrade VMP, Cardoso CL. Visitas Domiciliares de Agentes Comunitários de Saúde: Concepções de Profissionais e Usuários. Psico-USF 2017; 22(1):87-98.

33. Kebian LVA, Acioli S. A visita domiciliar de enfermeiros e agentes comunitários de saúde da Estratégia Saúde da Família. Rev Eletr Em 2014;16(1):161-169.

Artigo apresentado em 20/11/2020

Aprovado em 20/05/2021

Versão final apresentada em 22/05/2021

Editores-chefes: Romeu Gomes, Antônio Augusto Moura da Silva 